\title{
Constraint summation in phonological theory
}

\author{
Giorgio Magri ${ }^{1}$ and Benjamin Storme ${ }^{2}$ \\ ${ }^{1}$ CNRS and ${ }^{2}$ University of Lausanne
}

\section{Introduction}

The classical constraints used in phonology evaluate individual candidate pairs of an underlying form and a corresponding surface realization (Prince and Smolensky 1993/2004). Yet, some authors have extended this classical constraint toolkit through constraints that evaluate not a single candidate but a set of candidates simultaneously. In Dispersion Theory (DT; Flemming 2002, 2004, 2008), distinctiveness constraints penalize surface forms which are not sufficiently distinct. In the Optimal Paradigms model (OPM; Kenstowicz 1997; McCarthy 2005), Optimal Paradigm faithfulness constraints penalize dissimilarities among surface forms in an inflectional paradigm.

The addition of distinctiveness and OP faithfulness constraints to the classical constraint set raises a subtle technical problem: since classical constraints apply to individual candidates while distinctiveness and OP faithfulness constraints instead apply to sets of candidates, the classical constraints must be "lifted" from individual candidates to sets of candidates. A natural solution to this problem (and indeed the solution pursued in DT and the OPM) is to lift a classical constraint $\mathrm{C}$ from individual candidates to sets of candidates by summing constraint violations across the candidates in the set, as in (1).

(1)

$$
\begin{aligned}
& \text { Constraint summation assumption: } \\
& \begin{array}{l}
C(\{\text { candidate } 1, \text { candidate } 2, \text { candidate } 3 \ldots\})= \\
\quad=C(\text { candidate } 1)+C(\text { candidate } 2)+C(\text { candidate } 3)+\ldots
\end{array}
\end{aligned}
$$

Is this assumption of constraint summation (1) an innocuous technicality or does it have substantial typological implications? To zoom in on this question, let us suppose that the constraint set contains no distinctiveness or OP faithfulness constraints but only classical constraints. We then have two options. According to the classical approach, we can compute the optimal surface realization of each underlying form individually relative to the original classical constraints. Alternatively, we can compute the set of optimal surface realizations for all the underlying forms simultaneously relative to the summed version (1) of the classical constraints. Do the two approaches select the same surface realizations so that constraint summation is innocuous? Or do they lead to different surface realizations, whereby constraint summation alters the typological predictions of the classical constraints? The individual constraint violations $C$ (candidate 1$)$ and $C$ (candidate 2$)$ cannot be reconstructed from their sum $C$ (candidate 1$)+C$ (candidate 2$)$. One might thus intuitively expect that the assumption of constraint summation wipes away much of the information encoded by the classical constraints, profoundly altering their typological implications. Extending a result by Prince (2015), this paper shows that this pessimism is unwarranted. Before establishing the typological innocuousness of constraint summation in section 3, we review in section 2 the arguments for the constraint summation assumption (1) in DT and the OPM.

\section{Constraint summation in DT and the OPM}

2.1 Constraint summation in DT The constraint-based phonological literature assumes two classes of constraints. Faithfulness constraints measure the distance or discrepancy between an underlying form and its surface realization. Markedness constraints measure the phonotactic ill-formedness of a surface form. Both

\footnotetext{
* We would like to thank Alan Prince for very useful feedback. We also would like to thank Edward Flemming for calling our attention to McCarthy (2005) as another application of constraint summation. The research reported in this paper has been supported by a grant from the ANR (project title: 'The mathematics of segmental phonotactics').
}

(c) 2020 Giorgio Magri and Benjamin Storme

Proceedings of AMP 2019 
types of constraints thus look at a single underlying/surface form candidate pair at the time. Flemming (2002, 2004) argues that this classical toolkit is insufficient because it fails to capture typological generalizations where contrast plays a role (see also Liljencrants \& Lindblom 1972; Padgett 2009; Stanton 2017). We review here one of Flemming's arguments, based on the typology of systems of contrasts among voiceless, plain voiced, and prenasalized voiced stops (Flemming 2004:258-263). Many languages contrast voiceless stops [p, t, k] with plain voiced stops [b, d, g] (e.g. French; Tranel 1987). Yet there are also a few languages that prefer having prenasalized voiced stops $\left[{ }^{\mathrm{n}} \mathrm{b},{ }^{\mathrm{n}} \mathrm{d},{ }^{\mathrm{n}} \mathrm{g}\right]$ (instead of plain voiceless stops) contrast with voiceless stops (e.g. San Juan Colorado Mixtec; Iverson \& Salmons 1996). How could we derive such a language within classical constraint-based phonology?

Obviously, we would need a markedness constraint which penalizes plain voiced stops at the exclusion of prenasalized ones. Let us call this constraint *D. The intuition behind this constraint could be that voicing is harder to sustain in a plain voiced stop than in a prenasalized one because the nasal aperture facilitates voicing by preventing a fast pressure build up above the glottis (Ohala 1983). We assume that this constraint *D is "counterbalanced" by another markedness constraint ${ }^{* n} \mathrm{D}$ that instead penalizes prenasalized voiced stops at the exclusion of plain ones. The intuition behind this constraint would be that prenasalized stops are more effortful to produce because they 'require rapid raising of the velum to produce oral and nasal phases within the same stop' (Flemming, 2004:260). Finally, we consider a third markedness constraint *VTV which penalizes voiceless stops in intervocalic position. The constraint set is completed by two faithfulness constraints Ident(voice) and Ident(nas) that protect the underlying specifications for voicing and nasalization, respectively.

Following Flemming, we adopt throughout this section the Optimality Theoretic (OT; Prince \& Smolensky 1993/2004) implementation of constraint-based phonology, whose formalism is reviewed below in section 3. Tableaux (2a) and (2b) show how this approach can derive an inventory with contrastive voiceless stops and prenasalized voiced stops, as in San Juan Colorado Mixtec.

a. \begin{tabular}{|r||cc|ccc|}
\hline /ata/ & $* \mathrm{D}$ & Ident(voice) & Ident(nas) & ${ }^{* \mathrm{n}} \mathrm{D}$ & $* \mathrm{VTV}$ \\
\hline \hline 䟚 $[\mathrm{ata}]$ & & & & & $*$ \\
\hline$[\mathrm{ada}]$ & $* !$ & $*$ & & & \\
\hline$\left[\mathrm{a}^{\mathrm{n}} \mathrm{da}\right]$ & & $* !$ & $*$ & $*$ & \\
\hline
\end{tabular}

b.

\begin{tabular}{|c|c|c|c|c|}
\hline /ada/ & *D Ident(voice) & Ident(nas) & ${ }^{* \mathrm{n}} \mathrm{D}$ & *VTV \\
\hline [ata] & $* !$ & & & $*$ \\
\hline [ada] & $* !$ & & & \\
\hline u安 $\left[\mathrm{a}^{\mathrm{n}} \mathrm{da}\right]$ & & $*$ & $*$ & \\
\hline
\end{tabular}

However, Flemming notes that no language prefers prenasalized voiced stops to plain voiced stops in a context where voiceless stops are banned. For instance, intervocalic voiceless stops are never repaired through intervocalic prenasalization. The only attested repair is intervocalic voicing (e.g. Tümpisa Shoshone; Dayley 1989). Flemming argues that this fact is difficult to derive with only the faithfulness and markedness constraints made available by classical constraint-based phonology. In fact, as soon as ${ }^{*} \mathrm{D}$ is allowed to outrank ${ }^{* n} \mathrm{D}$, we derive an unattested pattern of intervocalic prenasalization of voiceless stops. This pattern is derived if *VTV and *D are top ranked, as shown in Tableau (3).

(3)

\begin{tabular}{|r||cc|ccc|}
\hline$/ \mathrm{ata} /$ & $* \mathrm{VTV}$ & $* \mathrm{D}$ & ${ }^{* \mathrm{n}} \mathrm{D}$ & Ident(voice) & Ident(nas) \\
\hline \hline$[\mathrm{ata}]$ & $* !$ & & & & \\
\hline$[\mathrm{ada}]$ & & $* !$ & & $*$ & \\
\hline 唃 $\left[\mathrm{a}^{\mathrm{d}} \mathrm{da}\right]$ & & & $*$ & $*$ & $*$ \\
\hline
\end{tabular}

These considerations suggest that our initial attempt at deriving the preference for prenasalized over plain voiced stops in Mixtec through classical markedness constraints is not on the right track. The constraint responsible for this preference cannot be a classical markedness constraint such as the constraint *D proposed above, because that constraint is blind to the presence or absence of a plain voiceless stop. This strategy based on classical markedness thus leads to the incorrect prediction that prenasalized voiced stops are preferred 
also in the absence of plain voiceless stops, namely that prenasalization can be used as a repair strategy for intervocalic voiceless stops.

In order to solve this impasse, Flemming proposes that the preference for prenasalized voiced stops in contexts where voiceless stops are available results from contrast enhancement: the voicing contrast is more distinct in the pair [t]-[ $\left.{ }^{\mathrm{n}} \mathrm{d}\right]$ than in the pair [t]-[d] (Iverson \& Salmons, 1996), due to the higher intensity of the periodic part of the speech signal in [ $\left.{ }^{\mathrm{n}} \mathrm{d}\right]$ than in [d]. In the presence of a voiceless stop, the preference for maximizing contrast can exert its effect and allow for [ $\left.{ }^{\mathrm{n}} \mathrm{d}\right]$ at the exclusion of plain [d]. But in the absence of voiceless stops, there is no contrast to enhance and thus the markedness of [ $\left.{ }^{\mathrm{n}} \mathrm{d}\right]$ relative to [d] is the only active force, whereby voiced stops are predicted to be systematically preferred.

Flemming formalizes the preference for more distinct phonological contrasts via a new family of constraints: distinctiveness constraints. Distinctiveness constraints penalize pairs of sounds based on their distance along a perceptual scale. In the case at hand, the relevant perceptual scale is the intensity of voicing. Following Flemming's simplifying assumption, suppose that the intensity of voicing is equal to 0 in voiceless stops, to 1 in plain voiced stops, and to 2 in prenasalized stops. Pairs [t]-[d] and [d]-[ $\left.{ }^{\mathrm{n}} \mathrm{d}\right]$ (but not $[\mathrm{t}]-\left[{ }^{\mathrm{n}} \mathrm{d}\right]$ ) violate a distinctiveness constraint requiring voicing contrasts corresponding to a distance strictly larger than one unit along the intensity scale. Let us refer to this constraint as MinDist, as in (4).

(4) MinDist

Assign a violation mark to pairs of surface forms with a voicing contrast corresponding to a distance equal to or smaller than 1 along the scale of voicing intensity.

Penalizes [t]-[d] and $[\mathrm{d}]-\left[{ }^{\mathrm{n}} \mathrm{d}\right]$ but not $[\mathrm{t}]-\left[{ }^{\mathrm{n}} \mathrm{d}\right]$.

Distinctiveness constraints are formally very different from classical faithfulness and markedness constraints. In fact, classical constraints assign a number of violations to each individual phonological mapping of an underlying form and a corresponding surface candidate. Distinctiveness constraints instead compare the surface realizations of multiple underlying forms based on their distinctiveness. This difference has implications for the architecture of grammar. A classical constraint-based grammar evaluates the candidates of a single underlying form at a time, as illustrated above with the two separate tableaux (2) for the two underlying forms /ata/ and /ada/. A grammar with distinctiveness constraints instead must evaluate sets of candidates corresponding to multiple underlying forms. But what about the classical constraints that are now mixed up with the new distinctiveness constraints? How can they be "lifted" from individual mappings to sets of mappings involving different underlying forms?

Flemming makes the natural suggestion that classical faithfulness and markedness constraints be redefined for sets of mappings by summing their constraint violations across all mappings in a set, as stated in (1). Tableau (5) illustrates how distinctiveness constraints and constraint summation of the classical constraints work in Dispersion Theory. We consider again the two underlying forms /ata/ and /ada/. This time, they occur together in the same tableau, rather than heading the two separate tableaux in (2). Since each of these two underlying forms comes with the three surface candidates [ata], [ada], and [a $\mathrm{a}^{\mathrm{n}} \mathrm{da}$, we need to consider $3 \times 3=9$ mappings, listed by row in (5). For instance, row (5d) corresponds to the (crazy) mapping whereby /ata/ is realized as [ada] and /ada/ as [ata].

(5)

\begin{tabular}{|c|c|c|c|c|c|c|}
\hline /ata/, /ada/ & $\frac{\vec{m}}{\vec{a}}$ & 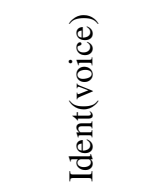 & 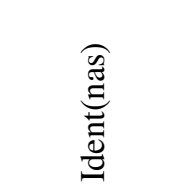 & $\stackrel{\vec{*}}{\stackrel{\oplus}{*}}$ & * & $\underset{*}{z}$ \\
\hline 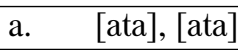 & & ${ }^{*} \mathrm{~d} \rightarrow \mathrm{t}$ & & & & $*_{\text {ata }}^{*}$ ata \\
\hline b. $\quad$ [ata], [ada] & $*_{\mathrm{t}-\mathrm{d}}$ & & & & $*_{d}$ & *ata \\
\hline c. $\quad[\mathrm{ata}],\left[\mathrm{a}^{\mathrm{n}} \mathrm{da}\right]$ & & & ${ }^{*} \mathrm{~d} \rightarrow \mathrm{n} \mathrm{d}$ & ${ }^{*}{ }_{\mathrm{n}} \mathrm{d}$ & $*_{d}$ & *ata \\
\hline d. $\quad[$ ada $],[$ ata $]$ & $*_{t-d}$ & ${ }_{t \rightarrow d}{ }^{*} d \rightarrow t$ & & & ${ }^{*} d$ & *ata \\
\hline $\begin{array}{ll}\text { e. } & \text { [ada], [ada] }\end{array}$ & & $*_{t} \rightarrow d$ & & & $*{ }^{*} * d$ & \\
\hline f. $\quad[\mathrm{ada}],\left[\mathrm{a}^{\mathrm{n}} \mathrm{da}\right]$ & ${ }^{*} \mathrm{~d}-{ }^{\mathrm{n}} \mathrm{d}$ & $*_{t \rightarrow d}$ & ${ }^{*} d \rightarrow{ }^{n} \mathrm{~d}$ & $*_{\mathrm{n}} \mathrm{d}$ & ${ }^{*} d^{*}{ }^{n} d$ & \\
\hline g. $\quad\left[\mathrm{a}^{\mathrm{n}} \mathrm{da}\right],[\mathrm{ata}]$ & & ${ }_{t \rightarrow}^{*} d^{*} d \rightarrow t$ & $*_{\mathrm{t} \rightarrow{ }^{\mathrm{n}} \mathrm{d}}$ & $*_{\mathrm{n}} \mathrm{d}$ & ${ }_{\mathrm{d}}$ & *ata \\
\hline h. $\left[\mathrm{a}^{\mathrm{n}} \mathrm{da}\right],[\mathrm{ada}]$ & ${ }^{*} \mathrm{~d}-{ }^{\mathrm{n}} \mathrm{d}$ & $*_{\mathrm{t} \rightarrow \mathrm{n}} \mathrm{d}$ & $*_{t \rightarrow}{ }^{n} \mathrm{~d}$ & ${ }^{*}{ }_{\mathrm{n}} \mathrm{d}$ & ${ }^{*} \mathrm{~d}^{* \mathrm{n}} \mathrm{d}$ & \\
\hline i. $\left[a^{n} d a\right],\left[a^{n} d a\right]$ & & $*_{t \rightarrow{ }^{n} \mathrm{~d}}$ & ${ }_{t \rightarrow}{ }^{n} d^{*} d \rightarrow{ }^{n} d$ & ${ }^{*} \mathrm{n}{ }^{*} \mathrm{n}_{\mathrm{n}}$ & ${ }^{*} \mathrm{n}{ }^{* \mathrm{n}} \mathrm{d}$ & \\
\hline
\end{tabular}


MinDist penalizes the set of mappings (5b), because it features two surface forms whose consonants sit on the voicing scale at a distance of 1 ([ata]-[ada]). It does not penalize the set of mappings (5c), because this set features two surface forms whose consonants sit on the voicing scale sufficiently far apart, namely at a distance of 2 ([ata]-[anda]). And so on. This constraint thus exerts a preference for the prenasalized over the plain voiced stop, although crucially only in the presence of the voiceless stop.

Flemming assumes that this MinDist contrast constraint is the only constraint that favors the prenasalized over the plain voiced stop, while classical markedness constraints prefer the plain over the prenasalized voiced stop. This means that we need to redefine the markedness constraint $* \mathrm{D}$ as penalizing all voiced stops, both plain and prenasalized ones. Thus, this constraint assigns two violations to the set of mappings (5f), because its two surface forms [ada] and [a $\mathrm{a}^{\mathrm{n}} \mathrm{da}$ ] both violate it and the two violations are summed together, as prescribed by the constraint summation assumption (1). Analogously, a classical faithfulness constraint such as Ident(voice) assigns two violations to the set of mappings (5d), because it assigns one violation to the mapping of /ata/ to [ada] and another violation to the mapping of /ada/ to [ata]. And so on. To make it easier to track constraint violations in the candidates, the specific pairs of output segments (in the case of distinctiveness constraints) and input-output segments (in the case of faithfulness constraints) and the specific segments/sequences (in the case of classical markedness constraints) that violate the corresponding constraint are indicated in subscript next to each violation mark.

This approach solves the problem discussed above: it easily derives a system contrasting voiceless stops and prenasalized voiced stops while blocking allophonic prenasalization of voiceless stops. In fact, a system with contrasting voiceless stops and prenasalized voiced stops is derived if MinDist is top ranked, as in (6).

\section{(6) $\quad$ MinDist $\gg \operatorname{Ident}\left(\right.$ voice), Ident(nasal), ${ }^{* n} \mathrm{D}, * \mathrm{D}, * \mathrm{VTV}$}

Ranking condition (6) has the effect of eliminating all languages that do not display the maximally dispersed $[\mathrm{t}]-\left[{ }^{\mathrm{n}} \mathrm{d}\right]$ contrast. Once these languages have been eliminated, there are two remaining languages: $\left\{\langle/\right.$ ata/, [ata] $\rangle,\left\langle/\right.$ ada $\left.\left./,\left[\mathrm{a}^{\mathrm{n}} \mathrm{da}\right]\right\rangle\right\}$ corresponding to row (5c) and $\left\{\left\langle/ \mathrm{ata} /,\left[\mathrm{a}^{\mathrm{n}} \mathrm{da}\right]\right\rangle,\langle/ \mathrm{ada} /,[\right.$ ata $\left.]\rangle\right\}$ corresponding to row $(5 \mathrm{~g})$. Among those, the first one will win under any ranking of the remaining constraints, as it violates a strict subset of the constraints violated by the second one. The two languages are equally bad in terms of markedness but $\left\{\langle/ \mathrm{ata} /,[\mathrm{ata}]\rangle,\left\langle/ \mathrm{ada} /,\left[\mathrm{a}^{\mathrm{n}} \mathrm{da}\right]\right\rangle\right\}$ involves strictly less violations of faithulness than $\left\{\left\langle/\right.\right.$ ata $\left./,\left[\mathrm{a}^{\mathrm{n}} \mathrm{da}\right]\right\rangle,\langle/$ ada $/,[$ ata $\left.]\rangle\right\}$, as shown in rows $(5 \mathrm{c})$ and $(5 \mathrm{~g})$.

As desired, the system does not derive intervocalic nasalization as a repair to intervocalic voiceless stops because the three logically possible languages that prenasalize intervocalic voiceless / $t$ / are all harmonically bounded. In fact, the language $\left\{\left\langle/ \mathrm{ata} /,\left[\mathrm{a}^{\mathrm{n}} \mathrm{da}\right]\right\rangle,\langle/ \mathrm{ada} /,[\mathrm{ata}]\rangle\right\}$ corresponding to row (5g) is harmonically bounded by the language $\left\{\langle/\right.$ ata $\left./,[\mathrm{ata}]\rangle,\left\langle/ \mathrm{ada} /,\left[\mathrm{a}^{\mathrm{n}} \mathrm{da}\right]\right\rangle\right\}$ corresponding to row $(5 \mathrm{c})$, as we just discussed. Furthermore, the language $\left\{\left\langle/ \mathrm{ata} /,\left[\mathrm{a}^{\mathrm{n}} \mathrm{da}\right]\right\rangle,\langle/ \mathrm{ada} /,[\mathrm{ada}]\rangle\right\}$ corresponding to row (5h) and the language $\left\{\left\langle/ a t a /,\left[a^{\mathrm{n}} d a\right]\right\rangle,\left\langle/ \mathrm{ada} /,\left[\mathrm{a}^{\mathrm{n}} \mathrm{da}\right]\right\rangle\right\}$ corresponding to row (5i) are both harmonically bounded by the language $\{\langle/$ ata $/,[$ ada $]\rangle,\langle/$ ada $/,[$ ada $]\rangle\}$ corresponding to row (5e).

2.2 Constraint summation in the OPM This section summarizes McCarthy's (2005) argument for constraint summation in the Optimal Paradigms model (OPM). Morphologically-related forms may bear resemblance that goes beyond what is predicted by the interaction of classical markedness constraints and input-output faithfulness constraints. A classical example is the case of the participle lightening [lartnin], where the stem-final consonant is realized as a syllabic nasal [n], as in the verb lighten [lartn], instead of the phonotactically expected [n]. Data of this kind have motivated positing another type of faithfulness besides input-output faithfulness: output-output faithfulness. ${ }^{1}$ Output-output faithfulness constraints enforce similarity among surface forms. In the case of surface inflected forms, similarity is enforced among surface forms in the same inflectional paradigm, i.e. forms that share a lexeme. In this approach, the presence of syllabic [n] in the participle lightening [lartnın] can be explained as the result of an output-output faithfulness constraint requiring similarity with the verb lighten [lartn] and outranking the input-output faithfulness constraint requiring similarity with the input stem /lartn/.

When one form in a paradigm is morphologically simpler than other paradigm members, this morphologically simpler form (i.e. the base) is always the one that other paradigm members must be faithful to (see Benua 1996:240-242 for a discussion of potential counterexamples). Lightening conforms to this

\footnotetext{
1 Output-output faithfulness is also motivated by patterns of reduplication (McCarthy \& Prince, 1995).
} 
generalization because it is asymmetrically influenced by its base lighten. In line with this generalization, theories of output-output faithfulness have been developed where the phonology of the base is computed in a first step and serves as input to the evaluation of affixed forms, alongside the affixed forms' underlying representations (e.g. Benua's 1996 Transderivational Correspondence Theory).

However, McCarthy (2005) notes that effects that can be analyzed as output-output faithfulness are also observed in paradigms where all forms are equally complex morphologically and where the choice of the attractor is not guided by morphological smiplicity or markedness but by phonological markedness. McCarthy (2005) illustrates his argument with Arabic verbal stems. In Arabic, verbal stems are required to end in VC (e.g. [faYal], [faSfal]). No stem ending in $\mathrm{V}: \mathrm{C}$ or $\mathrm{VCC}$ is attested in verbal paradigms (e.g. *[faYa:l], *[fail]). This contrasts with nominal stems, which can end in VC, V:C, and VCC (e.g. [faYal], [faYa:l], [fail]). Under Richness of the Base, the fact that the phonological shape of verbal stems is more constrained than that of nominal stems is unexpected.

McCarthy's insight is that this apparent quirk of Arabic verbs can be explained as an effect of outputoutput faithfulness, combined with an independent property that distinguishes nouns and verbs in Arabic. Nominal suffixes in Arabic all start with a vowel whereas verbal suffixes start with vowels or consonants (see McCarthy 2005:179-180 for a list of suffixes). In a nutshell, due to a high ranking markedness constraint banning super heavy syllables (*V:CCV, $\left.{ }^{*} \mathrm{VCCCV}\right)$, verbal stems followed by consonant-initial suffixes can only afford short vowels in stem-final syllables (e.g. [faCal-tu] but *[faSa:l-tu] and *[faSl-tu]). Output-output faithfulness then extends the short vowel that is phonotactically expected before consonant-initial suffixes to the whole paradigm, including to forms built with vowel-initial suffixes and where short vowels are not phonotactically required. In nouns, only vowel-initial suffixes are attested. Therefore, contrary to inflected verbs, there is no paradigmatic pressure to extend stems ending in $\mathrm{VC}$-, therefore allowing for all $\mathrm{VC}-, \mathrm{V}: \mathrm{C}-$, and VCC- to surface faithfully in inflected nouns.

For this analysis to be implemented using Benua's Transderivational Correspondence Theory, it is necessary to assume that the base in verbal paradigms is one of the forms built with a consonant-initial suffix. Faithfulness to the base then extends the short vowel that is phonotactically expected in this form to all other forms. Tableaux (7a) and (7b) show how this analysis works, focusing on two forms of the paradigm of hypothetical underlying /faYa:l/: (i) an inflected form with a consonant-initial suffix and that serves as the base in the paradigm, /fafa:l-tu/ (1st singular perfective), and (ii) an inflected form with a vowel-initial suffix, /fa9a:l-a/ ( $3 \mathrm{~d}$ singular perfective). To get the short vowel to be extended to other forms, /faAa:l-tu/ has to be considered as the base. As the base, its phonology is computed first. In this first cycle, only input-output correspondence is relevant. Because *V:CCV outranks the faithfulness constraint protecting underlying vowel length (IdentIO(length)), the stem long vowel is shortened before $\mathrm{CC}$, as shown in Tableau (7a). In a second step, the phonology of other paradigm members is computed. Now, output-output correspondence is relevant, with [faSa:ltu] serving as the base for the surface form derived from underlying /faSa:l-a/. IdentBD(length) requires the form under evaluation to match the base along vowel length. This constraint outranks IdentIO(length), therefore favoring base-derivative similarity over input-output similarity, as shown in Tableau (7b).

(7)

\begin{tabular}{|c|c|c|}
\hline /faYa:1-tu/ & *V:CCV IdentBD(length) & IdentIO(length) \\
\hline a. [faYa:ltu] & $* !$ & \\
\hline b. [faSaltu] & & * \\
\hline $\begin{array}{r}\text { /faYa:l-a/ } \\
\text { Base }=[\text { faSaltu }]\end{array}$ & *V:CCV IdentBD(length) & IdentIO(length) \\
\hline a. [faYa:la] & *! & \\
\hline b. [faiala] & & $*$ \\
\hline
\end{tabular}

As pointed out by McCarthy, the problem with this approach is that there is no independent, morphological motivation to treat a form with a consonant-initial suffix (/faSa:l-tu/ in our case) as the base. Indeed, inflected forms with consonant-initial suffixes are neither simpler than the others paradigm members (all forms are inflected) nor morphosyntactically less marked. Indeed, morphosyntactic markedness predicts that the third person singular form should be the base. However, all third person singular forms in the verbal paradigm are built with vowel-initial suffixes (cf. /faSa:l-a/ in our example). 
To solve this issue, McCarthy proposes Optimal Paradigm faithfulness constraints. Surface inflected forms are related by output-output correspondence to all other inflected forms of the same stem. The stem of every paradigm member stands in correspondence with the stem of other members. The constraint placing all stems of an inflectional paradigm in correspondence is Optimal Paradigm faithfulness. This is distinct from Benua's Transderivational Correspondence Theory because the latter is asymmetrical (the base is generated 'first', hence not modifiable) while the effects of OP faithfulness are symmetric: all members in a paradigm are evaluated simultaneously hence each of them can be modified.

In the case of Arabic, extension of the short vowel from stems built with consonant-initial suffixes is enforced by an OP faithfulness constraint that requires matching vowel length in all pairs of paradigm members, as defined in (8). This constraint evaluates surface resemblance symmetrically across inflectionally related forms, hence it does not stipulate that any paradigm member should be a priori preferred over the others. In a concrete analysis, the choice of the attractor is determined by markedness, as will be shown in more details below.

(8) Ident-OP(length)

In every paradigm, the stem of each paradigm member corresponds to the stem of every other paradigm member.

In each pair of correspondent stems S1-S2, assign a violation mark for each vowel in S1 that does not have the same length as the corresponding vowel in S2.

Penalizes paradigms $<$ faSa:l-ta, faSal- $\mathbf{u}>$ and $<$ faSal-ta, faSa:l-u $>$. Does not penalize paradigms $<$ faSal-ta, faSal-u $>$ and $<$ faSa:l-ta, faYa:l-u $>$

OP faithfulness constraints are formally very different from classical faithfulness and markedness constraints. In fact, classical constraints assign a number of violations to each individual candidate mapping of an underlying form and a corresponding surface realization. OP faithfulness constraints instead compare the surface realizations of multiple underlying forms based on their similarity. This difference has the same implications for the architecture of grammar as in the case of Dispersion Theory.

How do OP faithfulness constraint interact with classical faithfulness and markedness constraints? Like Flemming for DT, McCarthy (2005:173) makes the natural suggestion that classical faithfulness and markedness constraints be redefined for sets of candidates by summing their constraint violations across all candidates in a set. Tableau (9) illustrates how OP faithfulness constraints and constraint summation of the classical constraints work in the Optimal Paradigms model. We consider again the two underlying forms /faYa:l-a/ and /faYa:l-tu/. This time, they occur together in the same tableau, rather than heading the two separate tableaux in (7).

(9)

\begin{tabular}{|r||cc|c|}
\hline /faSa:l-a/, /faSa:l-tu/ & $* \mathrm{~V}: \mathrm{CCV}$ & IdentOP(length) & IdentIO(length) \\
\hline \hline a. [faYa:l-a], [faYa:l-tu] & $* !$ & & \\
b. [faSa:l-a], [faSal-tu] & & $*$ & $*$ \\
c. [faSal-a], [faYa:l-tu] & $* !$ & $*$ & $*$ \\
d. [faSal-a], [faSal-tu] & & & $* *$ \\
\hline
\end{tabular}

The Optimal Paradigm faithfulness constraint IdentOP(length) penalizes the sets of mappings (9b) and (9c), because they feature two vowels that stand in correspondence but do not match in length. This constraint thus exerts a preference for paradigm uniformity, without specifying which form will be the attractor: candidates (9a) and (9d) are equally good in terms of paradigm uniformity. The choice of the attractor is determined by the high ranking markedness constraint $* \mathrm{~V}: \mathrm{CCV}$, which penalizes the candidate with a super heavy syllable (9a).

In a nutshell, this approach is able to derive the generalization on verbal stems in Arabic without needing to stipulate that inflected forms with consonant-initial suffixes are the base, in the morphological sense of Benua. The reason why the short vowel length is extended to other forms rather than the long one is the fact that super heavy syllables are marked.

\section{Is constraint summation typologically innocuous?}

The preceding section has reviewed some phonological theories (such as DT and the OPM) that share two formal innovations. The first innovation is that the classical constraint set is enriched with constraints 
(such as distinctiveness and OP faithfulness constraints) that evaluate multiple candidates simultaneously by comparing their surface forms in terms of their distinctiveness or their mutual faithfulness. These constraints are therefore formally different from classical faithfulness and markedness constraints, that instead evaluate candidates individually, one at the time. The second related innovation is that classical markedness and faithfulness constraints are "lifted" from individual candidates to sets of multiple candidates through the constraint summation assumption (1). This paper takes a closer look at this constraint summation assumption. Is this assumption typologically innocuous? In other words, is it the case that constraint summation does not alter the typological implications of classical markedness and faithfulness constraints? Or is it instead the case that phonological theories (such as DT and the OPM) that make use of constraint summation predict very different typologies even when the constraint set consists only of classical constraints?

3.1 Preliminaries We consider two underlying forms (the extension to an arbitrary finite number of underlying forms is straightforward). In order to focus on the constraint summation assumption (1), we suppose that the constraint set consists of $n$ classical constraints $C_{1}, \ldots, C_{n}$, but contains no distinctiveness or OP faithfulness constraints. We denote by $\alpha$ the generic surface candidate of the first underlying form and we collect these surface candidates into a candidate set $A$. The classical constraints $C_{1}, \ldots, C_{n}$ assign to (the mapping of that underlying form into) the candidate $\alpha$ the $n$ constraint violations $a_{1}, \ldots, a_{n}$. We collect them together into a tuple $\mathbf{a}=\left(a_{1}, \ldots, a_{n}\right)$. Analogously, we denote by $\beta$ the generic surface candidate of the second underlying form and we collect these surface candidates into a candidate set $B$. The classical constraints $C_{1}, \ldots, C_{n}$ assign to (the mapping of that second underlying form into) the candidate $\beta$ a tuple $\mathbf{b}=\left(b_{1}, \ldots, b_{n}\right)$ of $n$ constraint violations $b_{1}, \ldots, b_{n}$. A concrete example of the sets $A$ and $B$ is provided by the two tableaux (2a) and (2b) for the two underlying forms /ata/ and /ada/. In this case, $n=5$, the candidate corresponding to the first row of the tableau $A$ is $\alpha=$ [ata], and the corresponding tuple of constraint violations is $\mathbf{a}=(0,0,0,0,1)$.

Under the assumption that the constraints suffice to capture all the relevant information, the optimal candidate for a given underlying form must be the one which violates the constraints the least, namely the one with the "smallest" tuple of constraint violations. To formalize this intuition, we extend the intuitive notion of "smaller than" from single numbers to tuples of numbers. Thus, the condition $\widehat{\mathbf{a}}<\mathbf{a}$ means that the tuple of constraint violations $\widehat{\mathbf{a}}$ is smaller than the tuple of constraint violations $\mathbf{a}$. Effectively, this means that we define an order < among tuples of constraint violations. Different implementations of (classical) constraint-based phonology considered in the literature differ for the choice of the partial order $<$ used to compare tuples of constraint violations. We denote by opt $t_{<} A$ and $o p t_{<} B$ the sets of optimal candidates of the candidate sets $A$ and $B$. Thus, a candidate $\widehat{\alpha}$ belongs to the set $o p t_{<} A$ provided there exists no other candidate $\alpha$ in $A$ such that the tuple a of constraint violations assigned to this candidate $\alpha$ is smaller than the tuple $\widehat{\mathbf{a}}$ of constraint violations assigned to the optimal candidate $\widehat{\alpha}$, namely $\mathbf{a}<\widehat{\mathbf{a}}$.

We note that the set opt $<A$ of optimal candidates can contain more than one candidate. In fact, two candidates $\widehat{\alpha}_{1}$ and $\widehat{\alpha}_{2}$ can both be optimal if their corresponding tuples of constraint violations $\widehat{\mathbf{a}}_{1}$ and $\widehat{\mathbf{a}}_{2}$ are incommensurable: neither of the two is larger than the other according to the order $<$ because $<$ is only partial. Furthermore, even if $<$ is total, two different candidates $\widehat{\alpha}_{1}$ and $\widehat{\alpha}_{2}$ can both be optimal if the constraints fail to distinguish between them, namely the two candidates share the same tuple $\widehat{\mathbf{a}}_{1}=\widehat{\mathbf{a}}_{2}$ of constraint violations.

We denote by $A \times B$ the collection of all pairs $(\alpha, \beta)$ of a candidate $\alpha$ from the candidate set $A$ and a candidate $\beta$ from the candidate set $B$. We "lift" the $n$ classical constraints from single candidates to pairs of candidates through the constraint summation assumption (1). This means that the lifted constraints assign to the candidate pair $(\alpha, \beta)$ the component-wise sum $\mathbf{a}+\mathbf{b}$ of the tuples $\mathbf{a}=\left(a_{1}, \ldots, a_{n}\right)$ and $\mathbf{b}=\left(b_{1}, \ldots, b_{n}\right)$ of constraint violations assigned to the two individual candidates $\alpha$ and $\beta$, as in (10).

$$
\mathbf{a}+\mathbf{b}=\left(a_{1}+b_{1}, \ldots, a_{k}+b_{k}, \ldots, a_{n}+b_{n}\right)
$$

In other words, $A \times B$ is the tableau that lists all pairs of candidates of the tableaux $A$ and $B$ and sums the stars in the two corresponding cells of $A$ and $B$. To illustrate, if $A$ and $B$ are the tableaux (2a) and (2b), their product $A \times B$ is the tableau (5) considered above stripped of the column corresponding to the distinctiveness constraint MinDist.

We denote by opt $_{<}(A \times B)$ the set of optimal candidate pairs of the candidate set $A \times B$. Thus, a candidate pair $(\widehat{\alpha}, \widehat{\beta})$ belongs to the set $\operatorname{opt}_{<}(A \times B)$ provided there exists no competing candidate pair 
$(\alpha, \beta)$ in $A \times B$ such that the sum $\mathbf{a}+\mathbf{b}$ of the two tuples $\mathbf{a}$ and $\mathbf{b}$ of constraint violations assigned to the two candidates $\alpha$ and $\beta$ is smaller than the sum $\widehat{\mathbf{a}}+\widehat{\mathbf{b}}$ of the two tuples $\widehat{\mathbf{a}}$ and $\widehat{\mathbf{b}}$ of constraint violations assigned to the two candidates $\widehat{\alpha}$ and $\widehat{\beta}$, namely $\mathbf{a}+\mathbf{b}<\widehat{\mathbf{a}}+\widehat{\mathbf{b}}$.

3.2 Typological innocuousness as a commutativity identity According to the classical implementation of constraint-based phonology, the order $<$ is used twice. It is used once to assign to the first underlying form a candidate $\widehat{\alpha}$ that individually violates the constraints the least, namely that belongs to the set $o p t_{<} A$ of optimal candidates of $A$. It is then used again and independently to assign to the second underlying form a candidate $\widehat{\beta}$ that also individually violates the constraints the least, namely that belongs to the set $o p t_{<} B$ of optimal candidates of $B$. According to phonological theories that make use of constraint summation (such as DT and the OPM), the order $<$ is instead used only once to assign to the two underlying forms considered a pair of surface candidates $(\widehat{\alpha}, \widehat{\beta})$ that jointly violate the constraints the least, namely a pair that belongs to the set opt $<(A \times B)$ of optimal pairs of $A \times B$, where candidate pairs are compared based on the sums of constraint violations of the two individual candidates, by virtue of the constraint summation assumption (1).

Suppose now that a candidate pair is optimal iff it consists of two optimal candidates, as stated in (11). This means that the two underlying forms considered end up with the same surface candidates no matter whether we adopt the classical approach or the approach based on constraint summation of DT and the OPM. In other words, the constraint summation assumption (1) made by DT and the OPM is typologically innocuous. And classical constraint-based phonology follows as a special case of DT and the OPM when the constraint set contains no distinctiveness or OP faithfulness constraints.

$$
\underbrace{\text { Commutativity identity: }}_{\begin{array}{c}
\text { constraint summation } \\
\text { approach (DT/OPM ) }
\end{array}}=\underbrace{o p t_{<} A \times o p t_{<} B}_{\text {classical approach }}
$$

In conclusion, a crucial issue of the formal analysis of phonological theories such as DT and the OPM is whether the identity (11) holds in the general case, for any two candidate sets $A$ and $B$. In other words, whether the two operations of optimization and product commute: by first combining candidates from $A$ and $B$ into pairs (through $\times$ ) and then optimizing (through opt $t_{<}$) over candidate pairs relative to the summed constraint violations (as prescribed by the left-hand side, which corresponds to the summationbased approach of DT or the OPM) we get the same result that we get by first optimizing (through $o p t_{<}$) within the two separate candidate sets $A$ and $B$ and then combining optimal candidates into pairs (through $\times$ ) (as prescribed by the right-hand side, which corresponds to classical constraint-based phonology).

3.3 Prince (2005)'s result Prima facie, there is every reason to be pessimistic about the validity of the commutativity identity (11). In fact, the sum $\mathbf{a}+\mathbf{b}$ of two tuples of constraint violations carries less information than the two individual tuples $\mathbf{a}$ and $\mathbf{b}$ : the individual tuples cannot be univocally reconstructed from the summed tuple. One might thus intuitively expect the commutativity identity (11) to fail because the assumption (1) of constraint summation can wipe away information encoded in the individual tuples of constraint violations which is crucial for optimization. Constraint summation would then not be typologically innocuous and classical constraint-based phonology could not be construed as a special case of DT and the OPM, even when the constraint set contains no distinctiveness or OP faithfulness constraints.

This pessimism is dispelled by a result from Prince (2015). He focuses on the special case where the order $<$ over tuples of constraint violations is OT's lexicographic order. Let us recall here the explicit definition of this order, that we have already used implicitly in section 2 . We start by linearly ordering or ranking the $n$ constraints $C_{1}, C_{2}, \ldots, C_{n}$ in some arbitrary way. Without loss of generality, we assume that constraint $C_{1}$ is ranked at the top, constraint $C_{2}$ is ranked right underneath it, and so on. The inequality $\mathbf{a}<\widehat{\mathbf{a}}$ then holds between any two tuples of constraint violations $\mathbf{a}=\left(a_{1}, \ldots, a_{n}\right)$ and $\widehat{\mathbf{a}}=\left(\widehat{a}_{1}, \ldots, \widehat{a}_{n}\right)$ provided there exists some $k \in\{1, \ldots, n\}$ which validates the conditions in (12). 


$$
\begin{aligned}
a_{1} & =\widehat{a}_{1} \\
& \vdots \\
a_{k-1} & =\widehat{a}_{k-1} \\
a_{k} & <\widehat{a}_{k}
\end{aligned}
$$

These conditions say that the $k-1$ top ranked constraints assign the same number of violations to the two candidates corresponding to the tuples a and $\widehat{\mathbf{a}}$ (these conditions are interpreted as vacuously true if $k=1$ ). And that the $k$ th constraint is then decisive because it assigns less violations to the candidate corresponding to the tuple a than to the candidate corresponding to the tuple $\widehat{\mathbf{a}}$. Constraints ranked underneath play no role.

Prince (2005) shows that the commutativity identity (11) holds for any two candidate sets $A$ and $B$ relative to OT's lexicographic order $<$ just recalled: a candidate $\widehat{\alpha}$ belongs to the set $o p t_{<} A$ of OT optimal candidates of $A$ and a candidate $\widehat{\beta}$ belongs to the set $o p t_{<} B$ of OT optimal candidates of $B$ if and only if the candidate pair $(\widehat{\alpha}, \widehat{\beta})$ belongs to the set $\operatorname{opt}_{<}(A \times B)$ of optimal candidate pairs of $A \times B$, when candidate pairs are compared based on summed constraint violations. In other words, despite constraint summation, theories such as DT and the OPM make the same typological predictions as classical OT when the constraint set only consists of classical constraints and no distinctiveness or OP faithfulness constraints, whereby constraint summation is typologically innocuous.

3.4 A simple proof of Prince's result Prince proves his result using a piece of notation specifically tailored to OT, namely elementary ranking conditions (ERCs; Prince 2002). But this line of reasoning turns out to be involved, intuitively because the operation of constraint summation does not admit a simple counterpart in the theory of ERCs. Yet, Prince's result admits an elementary explanation when we reason directly in terms of violation profiles rather than ERCs. To present our derivation of Prince's result, we split the commutativity identity (11) into the two inclusions (13) and consider them separately.

$$
\begin{array}{ll}
\text { a. } & \text { opt } \\
\text { b. } & \text { opt } \\
\text { b }(A \times B) \subseteq \text { opt } & A \times \text { opt } \\
\hline & \supseteq o p t_{<} A \times o p t_{<} B
\end{array}
$$

To establish the inclusion (13a), let us assume by contradiction that it fails. This means that a candidate pair $(\widehat{\alpha}, \widehat{\beta})$ is OT optimal in $A \times B$ but that, say, the candidate $\widehat{\alpha}$ is not OT optimal in $A$. This contradictory assumption means that there exists a different candidate $\alpha$ in $A$ that beats (has smaller constraint violations than) the candidate $\widehat{\alpha}$. In other words, the tuples $\mathbf{a}=\left(a_{1}, \ldots, a_{n}\right)$ and $\widehat{\mathbf{a}}=\left(\widehat{a}_{1}, \ldots, \widehat{a}_{n}\right)$ of constraint violations of the two candidates $\alpha$ and $\widehat{\alpha}$ satisfy the inequality $\mathbf{a}<\widehat{\mathbf{a}}$. This inequality means that conditions (12) hold for some $k \in\{1, \ldots, n\}$. By adding the corresponding components $\widehat{b}_{1}, \ldots, \widehat{b}_{k-1}, \widehat{b}_{k}$ of the tuple $\widehat{\mathbf{b}}$ of constraint violations of candidate $\widehat{\beta}$ to both sides of the inequalities (12), we obtain (14).

$$
\begin{aligned}
a_{1}+\widehat{b}_{1} & =\widehat{a}_{1}+\widehat{b}_{1} \\
& \vdots \\
a_{k-1}+\widehat{b}_{k-1} & =\widehat{a}_{k-1}+\widehat{b}_{k-1} \\
a_{k}+\widehat{b}_{k} & <\widehat{a}_{k}+\widehat{b}_{k}
\end{aligned}
$$

Conditions (14) in turn say that $\mathbf{a}+\widehat{\mathbf{b}}<\widehat{\mathbf{a}}+\widehat{\mathbf{b}}$. In other words, the candidate pair $(\alpha, \widehat{\beta})$ beats the candidate pair $(\widehat{\alpha}, \widehat{\beta})$. This conclusion contradicts the assumption that the candidate pair $(\widehat{\alpha}, \widehat{\beta})$ is OT optimal in $A \times B$.

The proof of the reverse inclusion (13b) is analogous. Indeed, let us assume by contradiction that the candidate $\widehat{\alpha}$ is OT optimal in $A$ and that the candidate $\widehat{\beta}$ is OT optimal in $B$ but that the pair $(\widehat{\alpha}, \widehat{\beta})$ is not OT optimal in $A \times B$. This means that there exists a different pair $(\alpha, \beta)$ in $A \times B$ such that $\mathbf{a}+\mathbf{b}<\widehat{\mathbf{a}}+\widehat{\mathbf{b}}$, where $\mathbf{a}, \mathbf{b}, \widehat{\mathbf{a}}, \widehat{\mathbf{b}}$ are the tuples of constraint violations of the four candidates $\alpha, \beta, \widehat{\alpha}, \widehat{\beta}$. Suppose that $\mathbf{a} \neq \widehat{\mathbf{a}}$. Since the lexicographic order $<$ is total and $\widehat{\alpha}$ is optimal in $A$, then $\widehat{\mathbf{a}}<\mathbf{a}$. This means that there exists $h$ such that $\widehat{a}_{1}=a_{1}, \ldots, \widehat{a}_{h-1}=a_{h-1}, \widehat{a}_{h}<a_{h}$. Analogously, suppose that $\mathbf{b} \neq \widehat{\mathbf{b}}$. Again, since $<$ is a total order and $\widehat{\beta}$ is optimal in $B$, then $\widehat{\mathbf{b}}<\mathbf{b}$. This means that there exists $k$ such that $\widehat{b}_{1}=b_{1}, \ldots, \widehat{b}_{k-1}=b_{k-1}, \widehat{b}_{k}<b_{k}$. Suppose without loss of generality that $h \geq k$. Thus $\widehat{a}_{1}+\widehat{b}_{1}=a_{1}+b_{1}, \ldots, \widehat{a}_{k-1}+\widehat{b}_{k-1}=a_{k-1}+b_{k-1}, \widehat{a}_{k}+$ $\widehat{b}_{k}<a_{k}+b_{k}$. This means that $\widehat{\mathbf{a}}+\widehat{\mathbf{b}}<\mathbf{a}+\mathbf{b}$, contradicting the assumption $\mathbf{a}+\mathbf{b}<\widehat{\mathbf{a}}+\widehat{\mathbf{b}}$. The cases where either $\mathbf{a}=\widehat{\mathbf{a}}$ or $\mathbf{b}=\widehat{\mathbf{b}}$ are treated analogously. 
3.5 Generalizing Prince's result beyond OT The reasoning developed in the preceding subsection to establish the commutativity identity (11) for OT rests on two properties of OT lexicographic orders. The first property is that the lexicographic ordering of two tuples of constraint violations is not affected by adding the same quantities to the constraint violations in the two tuples, whereby the inequalities (12) entail those in (14). To generalize this property, we say that a strict order $<$ is additive provided it satisfies condition (15) for any three tuples a, b, c of constraint violations (Anderson \& Feil 1988). Recall from (10) that "+" denotes the component-wise sum of two tuples of constraint violations.

$$
\begin{aligned}
\text { If: } & \mathbf{a}<\mathbf{b}, \\
\text { then: } & \mathbf{a}+\mathbf{c}<\mathbf{b}+\mathbf{c} .
\end{aligned}
$$

This implication (15) captures the intuition that, if $\mathbf{a}$ is smaller than $\mathbf{b}$ and if the same quantity $\mathbf{c}$ is added to both, the resulting sum $\mathbf{a}+\mathbf{c}$ ought to be smaller than the sum $\mathbf{b}+\mathbf{c}$. Although this additivity implication feels intuitive, it is possible to construct orders which flout it (see subsection 3.6 for a counterexample).

The second crucial property of OT lexicographic orders used in subsection 3.4 is that they are total. We want instead to allow for the possibility that the strict order $<$ is partial (as motivated in particular by the application to HG in the next subsection 3.6). This means that there can exist two tuples $\mathbf{a}$ and $\mathbf{b}$ of constraint violations such that neither $\mathbf{a}<\mathbf{b}$ nor $\mathbf{b}<\mathbf{a}$. In this case, we say that $\mathbf{a}$ and $\mathbf{b}$ are incommensurable (relative to $<$ ) and we write $\mathbf{a} \sim \mathbf{b}$. In other words, a strict partial order $<$ defines a corresponding incommensurability relation $\sim$. Since the strict order $<$ is irreflexive, the inequality $\mathbf{a}<\mathbf{a}$ fails for any tuple a of constraint violations. In other words, any tuple $\mathbf{a}$ is incommensurable with itself and the incommensurability relation $\sim$ is therefore reflexive. Furthermore, the incommensurability relation $\sim$ is obviously symmetric. The strict order $<$ is called weak provided the corresponding incommensurability relation $\sim$ is also transitive, namely qualifies as an equivalence relation among tuples of constraint violations (Roberts \& Tesman 2005:section 4.2.4). Weak orders generalize total orders in the following sense. According to a total order, any two tuples of constraint violations which are not ordered (neither is larger than the other) must be identical. In other words, the incommensurability relation defined by a total order is the identity relation. According to a weak order, tuples which are not ordered need not be identical but must be equivalent, in the sense that the incommensurability relation is an equivalence relation.

It turns out that Prince's result can be extended well beyond OT, as stated in (16). The "if" statement says that additive weak orders provide sufficient structure to ensure the commutativity identity (11). The "only if" statement says that additive weak orders also provide the necessary structure for the commutativity identity (11) to hold. In other words, the constraint summation assumption (1) made by DT and the OPM is typologically innocuous if and only if tuples of constraint violations are compared and optimized relative to an additive weak order.

$$
\text { A strict (possibly partial) order }<\text { over tuples of constraint violations satisfies the commutativity }
$$
identity (11) for any two candidate sets $A, B$ if and only if $<$ is an additive weak order.

The proof os statement (16) is a simple generalization of the reasoning used in subsection 3.4 for the special case of OT's lexicographic orders, as shown in Magri \& Storme (2020).

3.6 Application to $H G$ We consider a utility function $U$ which assigns to each tuple a of constraint violations a number $U(\mathbf{a})$. We can order the tuples of constraint violations based on their utility, with smaller tuples corresponding to a smaller utility, as in (17).

$$
\mathbf{a}<\mathbf{b} \text { if and only if } U(\mathbf{a})<U(\mathbf{b}) \text {. }
$$

The resulting relation $<$ is obviously a strict order. It is partial, because tuples of constraint violations which achieve the same utility are incommensurable. Furthermore, it is a weak order, because the corresponding incommensurability relation $\sim$ described in (18) is obviously transitive.

(18) $\mathbf{a} \sim \mathbf{b}$ if and only if $U(\mathbf{a})=U(\mathbf{b})$.

Suppose that the utility function $U$ is additive: the utility $U(\mathbf{a}+\mathbf{b})$ of the sum $\mathbf{a}+\mathbf{b}$ of any two tuples $\mathbf{a}, \mathbf{b}$ of constraint violations coincides with the sum $U(\mathbf{a})+U(\mathbf{b})$ of their utilities. In this case, the corresponding weak strict order $<$ satisfies the additivity implication (15). In fact, the antecedent $\mathbf{a}<\mathbf{b}$ of this additivity implication means that $U(\mathbf{a})<U(\mathbf{b})$. By adding the quantity $U(\mathbf{c})$ to both sides, we obtain $U(\mathbf{a})+U(\mathbf{c})<$ 
$U(\mathbf{b})+U(\mathbf{c})$. Because of the assumption that $U$ is additive, this inequality means in turn that $U(\mathbf{a}+\mathbf{c})<$ $U(\mathbf{b}+\mathbf{c})$. Finally, the latter inequality establishes the consequent $\mathbf{a}+\mathbf{c}<\mathbf{b}+\mathbf{c}$ of the additivity implication (15). The claim in (16) thus ensures that the commutativity identity (11) crucial for DT and the OPM holds whenever grammatical optimization is relative to the order induced by an additive utility function.

It turns out that for any additive utility function $U$, there exist $n$ weights $w_{1}, \ldots, w_{n}$ such that the utility $U(\mathbf{a})$ of any tuple $\mathbf{a}=\left(a_{1}, \ldots, a_{n}\right)$ of (integral) constraint violations $a_{1}, \ldots, a_{n}$ is the weighted sum of these constraint violations, namely $U(\mathbf{a})=\sum_{i=1}^{n} a_{i} w_{i}$. The proof of this statement is essentially the proof of the Fundamental Theorem of Linear Algebra (Strang 2006:section 2.6), whereby a linear application between finite dimensional spaces admits a matrix representation. The only twist is that we do not need linearity (namely additivity plus homogeneity) but additivity suffices, because we are only dealing with integral vectors (see Magri 2020). In other words, the partial strict order corresponding to an additive utility function in the sense of (17) yields the HG model of grammatical optimization (Legendre et al. 1990b,a; Smolensky \& Legendre 2006; Pater 2009; Potts et al. 2010). The claim in (16) thus ensures that the commutativity identity (11) crucial for DT and the OPM holds for any two candidate sets $A$ and $B$ relative to HG's order < corresponding to any constraint weighting.

Crucially, the claim in (16) provides not only a sufficient but also a necessary condition for the commutativity identity (11) to hold. Thus, this proposition can be used not only to verify that the commutativity identity holds, as we have done so far. But also to disprove that it does. To illustrate, suppose that there are only $n=2$ constraints and consider the quadratic utility function $U$ defined as in (19) for any pair $\mathbf{a}=\left(a_{1}, a_{2}\right)$ of constraint violations.

$$
U(\mathbf{a})=a_{1}^{2}+a_{2}^{2}
$$

The corresponding relation $<$ as in (17) is a weak partial strict order. Yet, it does not satisfy the additivity condition (15). In fact, consider for instance $\mathbf{a}=(2,2), \mathbf{b}=(0,3)$ and $\mathbf{c}=(4,4)$. In this case, $\mathbf{a}<\mathbf{b}$ (because $U(\mathbf{a})=2^{2}+2^{2}=8$ while $U(\mathbf{b})=0^{2}+3^{2}=9$ ). But $\mathbf{b}+\mathbf{c}<\mathbf{a}+\mathbf{c}$ (because $U(\mathbf{a}+\mathbf{c})=6^{2}+6^{2}=72$ while $\left.U(\mathbf{b}+\mathbf{c})=4^{2}+7^{2}=65\right)$. The claim in (16) therefore ensures that the commutativity identity (11) crucial for DT and the OPM fails when grammatical optimization is relative to the order induced by the quadratic utility function (19).

\section{Conclusions}

Usually in the constraint-based phonological literature (starting with Prince \& Smolensky 1993/2004), each underlying form comes with a preassigned set of candidates. Each of these candidates is represented as a tuple of constraint violations. These tuples are compared according to some strict (possibly partial) order $<$ that extends from single numbers to tuples of numbers the notion of "being smaller than". The best candidate for a given underlying form is the one which violates the constraints the least, namely the one with the smallest tuple of constraint violations.

DT (Flemming 2002, 2004, 2008) enriches the classical constraint set with distinctiveness constraints. And approaches to paradigm uniformity effects such as the OPM (Kenstowicz 1997; McCarthy 2005) enrich the classical constraint set with OP faithfulness constraints. Crucially, distinctiveness and OP faithfulness constraints evaluate not a single candidate but multiple candidates simultaneously, as they compare multiple candidate surface forms relative to their contrastiveness and their similarity, respectively. As a consequence, classical (faithfulness and markedness) constraints need to be "lifted" from individual candidates to sets of candidates. A reasonable way to do that is to sum their violations across multiple candidates.

What are the typological implications of this assumption of constraint summation made by DT and the OPM? We have formulated this question explicitly as follows. Suppose that we restrict ourselves to a constraint set which includes no distinctiveness or OP faithfulness constraints but only classical (markedness and faithfulness) constraints. In this case, can we guarantee that the typological predictions of DT and the OPM coincide with those of the classical theory, despite constraint summation? In other words, is the assumption of constraint summation made by DT and the OPM typologically innocuous?

This paper has shown that constraint summation is indeed typologically innocuous when constraint optimization is performed relative to an order $<$ of tuples of constraint violations which is additive and weak. And that additive weak orders provide the minimal structure for typological innocuousness to hold. This technical condition on grammatical optimization is verified for instance in the case of OT and HG. Our 
result extends and systematizes an earlier independent result for OT obtained in Prince (2015). This result provides a solid foundation for theories such as DT and the OPM which make use of constraint summation, for a large class of modes of constraint interaction.

\section{References}

Anderson, Marlow \& Todd Feil (1988). Lattice Ordered Groups: an Introduction. D. Reidel Publishing Company.

Benua, Laura (1996). Case studies in transderivational identity i: Javanese. University of Massachusetts.

Dayley, Jon P. (1989). Tümpisa (Panamint) Shoshone grammar. University of California Press, Berkley.

Flemming, Edward (2004). Contrast and perceptual distinctiveness. Hayes, Bruce, Robert Kirchner \& Donca Steriade (eds.), Phonetically-Based Phonology, Cambridge University Press, 232-276.

Flemming, Edward (2008). The realized input. Unpublished manuscript, MIT.

Flemming, Edward S (2002). Auditory representations in phonology. Routledge.

Iverson, Gregory K. \& Joseph C. Salmons (1996). Mixtec prenasalization as hyper-voicing. International Journal of American Linguistics 62, 165-175.

Kenstowicz, Michael (1997). Base identity and uniform exponence: alternatives to cyclicity. Durand, Jacques \& Bernard Laks (eds.), Current Trends in Phonology: Models and Methods, Salford: University of Salford, 363-394.

Legendre, Géraldine, Yoshiro Miyata \& Paul Smolensky (1990a). Harmonic Grammar: A formal multi-level connectionist theory of linguistic well-formedness: An application. Gernsbacher, Morton Ann \& Sharon J. Derry (eds.), Proceedings of the 12th annual conference of the Cognitive Science Society, Lawrence Erlbaum Associates, Hillsdale, NJ, 884-891.

Legendre, Géraldine, Yoshiro Miyata \& Paul Smolensky (1990b). Harmonic Grammar: A formal multi-level connectionist theory of linguistic well-formedness: Theoretical foundations. Gernsbacher, Morton Ann \& Sharon J. Derry (eds.), Proceedings of the 12th annual conference of the Cognitive Science Society, Lawrence Erlbaum, Hillsdale, NJ, 388-395.

Liljencrants, Johan \& Björn Lindblom (1972). Numerical simulation of vowel quality systems: The role of perceptual contrast. Language 48, 839-862.

Magri, Giorgio (2020). A principled derivation of Harmonic Grammar. Ettinger, Allyson, Gaja Jarosz \& Max Nelson (eds.), Proceedings of the third meeting of the Society for Computation in Linguistics, Association for Computational Linguistics, -.

Magri, Giorgio \& Benjamin Storme (2020). Constraint summation in phonological theory. To appear in the Journal of Language Modeling.

McCarthy, John J. (2005). Optimal paradigms. Downing, Laura J., T. Alan Hall \& Renate Raffelsiefen (eds.), Paradigms in Phonological Theory, Oxford University Press.

McCarthy, John J. \& Alan Prince (1995). Faithfulness and reduplicative identity. Beckman, Jill, Suzanne Urbanczyk \& Laura Walsh Dickey (eds.), University of Massachusetts Occasional Papers in Linguistics 18: Papers in Optimality Theory, GLSA, Amherst, 249-384.

Ohala, John J. (1983). The origin of sound patterns in vocal tract constraints. MacNeilage, Peter F. (ed.), The production of speech, Springer-Verlag, New York, 189-216.

Padgett, Jaye (2009). Systemic contrast and Catalan rhotics. The Linguistic Review 26, 431-463.

Pater, Joe (2009). Weighted constraints in Generative Linguistics. Cognitive Science 33, 999-1035.

Potts, Christopher, Joe Pater, Karen Jesney, Rajesh Bhatt \& Michael Becker (2010). Harmonic Grammar with Linear Programming: From linear systems to linguistic typology. Phonology 27(1), 1-41.

Prince, Alan (2002). Entailed ranking arguments. Manuscript (Rutgers University). Available from the Rutgers Optimality Archive as ROA 500.

Prince, Alan (2015). One tableau suffices. Manuscript (Rutgers University). Available from the Rutgers Optimality Archive as ROA 1250.

Prince, Alan \& Paul Smolensky (1993/2004). Optimality Theory: Constraint Interaction in generative grammar. Blackwell, Oxford, URL http://roa.rutgers.edu. Original version, Technical Report CU-CS-696-93, Department of Computer Science, University of Colorado at Boulder, and Technical Report TR-2, Rutgers Center for Cognitive Science, Rutgers University, April 1993. Available from the Rutgers Optimality Archive as ROA 537.

Roberts, Fred S. \& Barry Tesman (2005). Applied Combinatorics. Taylor and Francis Group.

Smolensky, Paul \& Géraldine Legendre (2006). The Harmonic Mind. MIT Press, Cambridge, MA.

Stanton, Juliet (2017). Constraints on the distribution of nasal-stop sequences: an argument for contrast. Ph.D. thesis, MIT.

Strang, Gilbert (2006). Linear Algebra and its applications. Thomson Brooks/Cole.

Tranel, Bernard (1987). The sounds of French: An introduction. Cambridge University Press. 